\title{
Energy Management and Peer-to-peer Trading in Future Smart Grids: A Distributed Game-Theoretic Approach
}

\author{
Giuseppe Belgioioso, Wicak Ananduta, Sergio Grammatico and Carlos Ocampo-Martinez
}

\begin{abstract}
We consider the economic dispatch problem for a day-ahead, peer-to-peer (P2P) electricity market of prosumers (i.e., energy consumers who can also produce electricity) in a distribution network. In our model, each prosumer has the capability of producing power through its dispatchable or nondispatchable generation units and/or has a storage energy unit. Furthermore, we consider a hybrid main grid \& P2P market in which each prosumer can trade power both with the main grid and with (some of) the other prosumers. First, we cast the economic dispatch problem as a noncooperative game with coupling constraints. Then, we design a fully-scalable algorithm to steer the system to a generalized Nash equilibrium (GNE). Finally, we show through numerical studies that the proposed methodology has the potential to ensure safe and efficient operation of the power grid.
\end{abstract}

\section{INTRODUCTION}

In recent years, environmental and sustainability concerns urged the pursuit of a novel energy system and electric power grid in which energy production, transmission, distribution, and consumption are more efficient and largely based on renewable sources, such as wind and solar power [1].

As a response to these challenges, the development of smart, sustainable, and green solutions is becoming more significant, including the sprouting of distributed energy resources, such as micro-generators (e.g., solar panels), storage units, and flexible loads, and the opening of energy markets. Simultaneously, the widespread deployment of sensing, information, and communication technology provides the means for efficiently manage the energy resources within the electricity grid through the establishment of various smart energy services, such as demand-side management and economic dispatch [2], [3].

Energy management mechanisms are considered as an increasingly essential element for implementing the smart grid paradigm and balancing massive energy production from renewable sources. In particular, a day-ahead economic dispatch process allows energy prosumers to efficiently manage their electricity production and consumption as well as

G. Belgioioso is with the Control Systems group, TU Eindhoven, The Netherlands. W. Ananduta and C. Ocampo-Martinez are with Institut de Robòtica i Informàtica Industrial (CSIC-UPC), Barcelona, Spain. S. Grammatico is with the Delft Center for Systems and Control (DCSC), TU Delft, The Netherlands. E-mail addresses: g.belgioiosodtue.nl, s.grammatico@tudelft.nl, \{wananduta, cocampo\}eiri.upc.edu. This work was partially supported by NWO under research projects OMEGA (grant n. 613.001.702), P2P-TALES (grant n. 647.003.003), the ERC under research project COSMOS (802348), the European Union's Horizon 2020 research and innovation programme under the Marie Skłodowska-Curie grant agreement No 675318 (INCITE). provides the main grid with an estimation of the amount of energy to be delivered over the upcoming day. To model and design energy management algorithms for the smart grid, game theory has been recently, yet extensively, adopted [4], [5]. Each prosumer (player, agent) in the day-ahead market (game) aims to select an energy schedule (decision variable) that minimizes its economic expense (cost function), which also depends on an aggregate measure of the schedules of the other prosumers. The latter is typically imposed to mitigate peak demand loads. Since the prosumers are, in general, selfinterested parties, a usual market equilibrium is considered to be the celebrated generalized Nash equilibrium (GNE).

In the control systems community, the problem of designing solution methods for multi-agent equilibrium problems in noncooperative networked games has recently gained high research interest. A fast-growing literature has been in fact developing decentralized algorithms for GNE seeking in noncooperative games with coupling constraints, e.g. [6]-[11] In turn, these methodologies provide the means to design more efficient and robust energy management mechanisms.

Although power systems are evolving towards a more decentralized management, electricity markets still perform resource allocation and pricing based on the conventional hierarchical and top-down approach [12] of power system management, which makes prosumers behave as passive receivers [13]. Actively incorporating prosumers in the electricity market by enabling peer-to-peer (P2P) energy tradings would instead allow for a bottom-up approach that empowers prosumers. In [14], [15], market models that involve P2P tradings are thought of as potential models due to the increasing of prosumers in the network. Moreover, a couple of $\mathrm{P} 2 \mathrm{P}$ energy markets have existed in the Netherlands and the United Kingdom [15]. However, the P2P paradigm poses a significant challenge in terms of modeling the decisionmaking process of the participants due to their conflicting interests [1]. In the literature, P2P energy trading mechanisms are proposed in various forms, e.g., matching contracts [16], consensus-based optimization [17]. Nevertheless, a very efficient algorithm to solve the resource allocation problem in a P2P market of prosumers is yet to be found.

In this paper, we consider a hybrid main grid \& $P 2 P$ electricity market formulation, in which each prosumer has generation and/or storage capabilities and can trade electricity both with the main grid and (some of) the other prosumers in the network. We show that the resulting resource allocation problem can be modeled as a noncooperative game with coupling constraints, representing the reciprocity of the P2P 
tradings and the limits of the aggregate load. Then, we propose a scalable and distributed algorithm, with convergence guarantee, to steer the decisions of the prosumers towards a GNE. We show through numerical simulations that the proposed methodology guarantees an efficient and safe operation of the system since reduces the overall economic expenses while ensuring the reciprocity of the tradings and the fulfillment of the grid limits.

Basic notation: $\mathbb{R}$ denotes the set of real numbers, $\mathbb{N}$ denotes the set of natural numbers, and 0 (1) denotes a matrix/vector with all elements equal to 0 (1); to improve clarity, we may add the dimension of these matrices/vectors as subscript. $A \otimes B$ denotes the Kronecker product between the matrices $A$ and $B$. For a square matrix $A \in \mathbb{R}^{n \times n}$, its transpose is $A^{\top},[A]_{i, j}$ represents the element on the row $i$ and column $j . A \succ 0(\succeq 0)$ stands for positive definite (semidefinite) matrix. Given $N$ vectors $x_{1}, \ldots, x_{N} \in \mathbb{R}^{n}$, $\boldsymbol{x}:=\operatorname{col}\left(x_{1}, \ldots, x_{N}\right)=\left[x_{1}^{\top}, \ldots, x_{N}^{\top}\right]^{\top}$.

Operator theoretic definitions: $\operatorname{Id}(\cdot)$ denotes the identity operator. The mapping $\iota_{S}: \mathbb{R}^{n} \rightarrow\{0, \infty\}$ denotes the indicator function for the set $\mathcal{S} \subseteq \mathbb{R}^{n}$, i.e., $\iota_{S}(x)=0$ if $x \in S, \infty$ otherwise. For a closed set $S \subseteq \mathbb{R}^{n}$, the mapping $\operatorname{proj}_{S}: \mathbb{R}^{n} \rightarrow S$ denotes the projection onto $S$, i.e., $\operatorname{proj}_{S}(x)=\operatorname{argmin}_{y \in S}\|y-x\|$. The set-valued mapping $\mathrm{N}_{S}: \mathbb{R}^{n} \rightrightarrows \mathbb{R}^{n}$ denotes the normal cone operator for the the set $S \subseteq \mathbb{R}^{n}$, i.e., $\mathrm{N}_{S}(x)=\varnothing$ if $x \notin S$, $\left\{v \in \mathbb{R}^{n} \mid \sup _{z \in S} v^{\top}(z-x) \leq 0\right\}$ otherwise. A set-valued mapping $\mathcal{F}: \mathbb{R}^{n} \rightrightarrows \mathbb{R}^{n}$ is (strictly) monotone if $(u-v)^{\top}(x-$ $y) \geq(>) 0$ for all $x \neq y \in \mathbb{R}^{n}, u \in \mathcal{F}(x), v \in \mathcal{F}(y)$.

\section{PRoblem Formulation}

In this section, the model of networked prosumers and the economic dispatch problem of each prosumer are presented.

\section{A. Peer-to-peer prosumer market model}

We model a day-ahead peer-to-peer electricity market of prosumers (agents) in a distribution network as an economic dispatch problem. Each agent might have the capability of producing power through its dispatchable or nondispatchable generation units and might also have a storage energy unit. Furthermore, we also consider that each agent might trade power with the main grid and might also trade power with some of the other agents, which are classified as the neighbors. Note that the neighbors of an agent might be defined based on geographical location of the agents or based on bilateral contracts that the agent has with the other agents [13]. The economic dispatch model is as follows.

Communication network: Let the set of agents be denoted by $\mathcal{N}=\{1,2, \ldots, N\}$. The communication among the agents is described by an undirected graph $\mathcal{G}=(\mathcal{N}, \mathcal{E})$, where $\mathcal{N}$ is the set of vertices (agents) and $\mathcal{E} \subseteq \mathcal{N} \times \mathcal{N}$ is the set of edges, with $|\mathcal{E}|=E$. The unordered pair of vertices $(i, j) \in \mathcal{E}$ if and only if agents $j$ and $i$ can exchange information. The set of neighbors of agent $i$ is defined as $\mathcal{N}_{i}=\{j \mid(j, i) \in \mathcal{E}\}$. Moreover, we consider Assumption 1.

Assumption 1: The graph $\mathcal{G}$ is connected.
Decision variables: For each agent $i \in \mathcal{N}$, denote by $p_{i, h}^{\mathrm{d}} \in \mathbb{R}$ the difference between the aggregate load at time instant $h$ and the total power produced by the non-dispatchable generation units owned by agent $i$. Note that positive $p_{i, h}^{\mathrm{d}}$ implies the aggregate load is larger than the power produced by agent $i$. Furthermore, denote the set of decisions at time $h$ by $u_{i, h}=\operatorname{col}\left(p_{i, h}^{\mathrm{dg}}, p_{i, h}^{\mathrm{st}}, p_{i, h}^{\mathrm{mg}},\left\{p_{(i, j), h}^{\mathrm{tr}}\right\}_{j \in \mathcal{N}_{i}}\right) \in \mathbb{R}^{n_{i}}$, where $p_{i, h}^{\mathrm{dg}} \in \mathbb{R}_{\geq 0}, p_{i, h}^{\mathrm{st}} \in \mathbb{R}, p_{i, h}^{\mathrm{mg}} \in \mathbb{R}$, and $p_{(i, j), h}^{\mathrm{tr}} \in \mathbb{R}$ are the total power produced by dispatchable generation units $(\mathrm{dg})$, the power delivered by/to the storage unit (st), the power traded with the main grid $(\mathrm{mg})$, and the power traded with neighbor $j \in \mathcal{N}_{i}$ (tr).

In this market, each agent aims at minimizing the cost of power usage to meet the load while satisfying some constraints imposed on the decision variables. The objective function and constraints associated with each decision variable are defined as follows:

Local power balance: Each agent has a power balance constraint as follows:

$$
\mathbf{1}_{n_{i}}^{\top} u_{i, h}=p_{i, h}^{\mathrm{d}} .
$$

Dispatchable generation units: One of the management objectives is to minimize the total power production of the dispatchable generation units, denoted by $p_{i, h}^{\mathrm{dg}}$. A typical class of cost functions for power production is of convex quadratic nature [18], [19], which here is denoted by $f_{i, h}^{\mathrm{dg}}$ and defined as follows:

$$
f_{i, h}^{\mathrm{dg}}(\cdot)=q_{i}^{\mathrm{dg}}(\cdot)^{2}+c_{i}^{\mathrm{dg}} \cdot
$$

where $q_{i}^{\mathrm{dg}}>0$ and $c_{i}^{\mathrm{dg}}$ are constants. Furthermore, the decision $p_{i, h}^{\mathrm{dg}}$ is constrained by

$$
\begin{aligned}
\underline{p}_{i}^{\mathrm{dg}} \leq p_{i, h}^{\mathrm{dg}} & \leq \bar{p}_{i}^{\mathrm{dg}}, & & \text { if } i \in \mathcal{N}^{\mathrm{dg}}, \\
p_{i, h}^{\mathrm{dg}} & =0, & & \text { otherwise },
\end{aligned}
$$

where $\bar{p}_{i}^{\mathrm{dg}}>\underline{p}_{i}^{\mathrm{dg}} \geq 0$ denote the maximum and minimum total power production of the dispatchable generation units, and $\mathcal{N}^{\mathrm{dg}} \subseteq \mathcal{N}$ denotes the set of agents that own dispatchable generation units.

a) Storage unit: Similarly to the dispatchable generation production, each agent might also minimize the usage of its storage unit, for instance, in order to maintain its longevity. The cost function is denoted by $f_{i, h}^{\text {st }}$ and defined as follows:

$$
f_{i, h}^{\mathrm{st}}\left(p_{i, h}^{\mathrm{st}}\right)=q_{i}^{\mathrm{st}}\left(p_{i, h}^{\mathrm{st}}\right)^{2}+c_{i}^{\mathrm{st}} p_{i, h}^{\mathrm{st}},
$$

where $q_{i}^{\text {st }} \geq 0$ and $c_{i}^{\text {st }}$ are constants. Moreover, the decision $p_{i, h}^{\mathrm{st}}$ is constrained by

$$
\left\{\begin{array}{r}
x_{i, h+1}=a_{i} x_{i, h}+b_{i} p_{i, h}^{\mathrm{st}}, \\
\underline{x}_{i} \leq x_{i, h+1} \leq \bar{x}_{i}, \quad \text { if } i \in \mathcal{N}^{\mathrm{st}} \\
-p_{i}^{\mathrm{ch}} \leq p_{i, h}^{\mathrm{st}} \leq p_{i}^{\mathrm{dh}}, \\
p_{i, h}^{\mathrm{st}}=0, \quad \text { otherwise }
\end{array}\right.
$$

where $x_{i, h}>0$ denotes the state of charge (SoC) of the storage unit, $a_{i} \in(0,1]$ denotes the efficiency of the storage and $b_{i}=-\frac{T_{\mathrm{s}}}{e_{\mathrm{cap}, i}}$, where $T_{\mathrm{s}}$ and $e_{\mathrm{cap}, i}$ denote the sampling 
time and the maximum capacity of the storage, respectively. Moreover, $\underline{x}_{i}, \bar{x}_{i} \in[0,1]$ denote the minimum and the maximum SoC of the storage of microgrid $i$, respectively whereas $p_{i}^{\text {ch }} \geq 0$ and $p_{i}^{\text {dh }} \geq 0$ denote the maximum charging and discharging power of the storage. Additionally, $\mathcal{N}^{\text {st }} \subseteq \mathcal{N}$ denotes the set of agents that own a storage unit.

Power traded with neighbors: Since agent $i$ can trade electricity with its neighbors, the trading cost is indicated by a linear function, denoted by $f_{i, h}^{\mathrm{tr}}$, as follows:

$$
f_{i, h}^{\mathrm{tr}}\left(\left\{p_{(i, j), h}^{\mathrm{tr}}\right\}_{j \in \mathcal{N}_{i}}\right)=\sum_{j \in \mathcal{N}_{i}} c_{(i, j)}^{\mathrm{tr}} p_{(i, j), h}^{\mathrm{tr}},
$$

where $c_{(i, j)}^{\mathrm{tr}}>0$ is the per-unit cost of electricity that is agreed between agent $i$ and $j$. In practice, the parameters $c_{(i, j)}^{\mathrm{tr}}$ can be agreed through a bilateral contract [14] or model taxes to encourage the development of certain technologies [20]. The power traded at time $h, p_{(i, j), h}^{\mathrm{tr}}$, is constrained by

$$
\begin{aligned}
-\bar{p}_{(i, j)}^{\operatorname{tr}} \leq p_{(i, j), h}^{\operatorname{tr}} & \leq \bar{p}_{(i, j)}^{\operatorname{tr}}, & & \forall j \in \mathcal{N}_{i}, \\
p_{(i, j), h}^{\operatorname{tr}}+p_{(j, i), h}^{\operatorname{tr}} & =0, & & \forall j \in \mathcal{N}_{i},
\end{aligned}
$$

where $\bar{p}_{(i, j)}^{\mathrm{tr}}$ denotes the maximum power can be traded with neighbor $j$. Moreover, (8) is called the reciprocity constraints [13], which couples the decisions of two neighboring agents. Note that each pair of neighboring agent $i$ and $j$ has the same reciprocity constraint.

Constraints of power traded with the main grid: As in [5], we assume that the electricity price depends on the total consumption of the network of prosumers and is typically defined as a quadratic function, i.e.,

$$
c_{h}^{\mathrm{mg}}\left(\hat{p}_{h}^{\mathrm{mg}}\right)=q_{h}^{\mathrm{mg}}\left(\hat{p}_{h}^{\mathrm{mg}}\right)^{2},
$$

where $\hat{p}_{h}^{\text {mg }}$ denotes the aggregate load on the main grid, i.e.,

$$
\hat{p}_{h}^{\mathrm{mg}}=\sum_{i \in \mathcal{N}} p_{i, h}^{\mathrm{mg}}
$$

and $q_{h}^{\mathrm{mg}}>0$ is a constant. Therefore, the objective function of agent $i$ associated to the trading with the main grid, denoted by $f_{i, h}^{\mathrm{mg}}$, is defined as

$$
\begin{aligned}
f_{i, h}^{\mathrm{mg}}\left(p_{i, h}^{\mathrm{mg}}, \hat{p}_{h}^{\mathrm{mg}}\right) & =c_{h}^{\mathrm{mg}}\left(\hat{p}_{h}^{\mathrm{mg}}\right) \frac{p_{i, h}^{\mathrm{mg}}}{\sum_{j \in \mathcal{N}} p_{j, h}^{\mathrm{mg}}} \\
& =q_{h}^{\mathrm{mg}}\left(\sum_{j \in \mathcal{N}} p_{j, h}^{\mathrm{mg}}\right) p_{i, h}^{\mathrm{mg}}
\end{aligned}
$$

Moreover, we impose that the total power that can be traded between all the agents and the main grid is also bounded, as follows:

$$
\underline{p}^{\mathrm{mg}} \leq \sum_{i \in \mathcal{N}} p_{i, h}^{\mathrm{mg}} \leq \bar{p}^{\mathrm{mg}}
$$

where $\bar{p}^{\mathrm{mg}}>\underline{p}^{\mathrm{mg}} \geq 0$ denote the upper and lower bounds. Note that the lower bound might be required to be positive in order to ensure a continuous operation of the main generators that supply the main grid.

\section{B. Economic dispatch problem}

Next, we introduce a compact notation for the decision variables and some of the constraints. In particular, the local constraints of agent $i$ that only involve its own decision variables, i.e.,

$$
u_{i, h} \in \mathcal{U}_{i, h}
$$

where $\mathcal{U}_{i, h}$ is a set such that (1), (3), (5), and (7) hold. Additionally, we define for all $i \in \mathcal{N}$ the stacked vector

$$
u_{i}=\operatorname{col}\left(\left\{u_{i, h}\right\}_{h \in \mathcal{H}}\right), \text { where } \mathcal{H}=\{1,2, \ldots, H\}
$$

denotes the set of time slots with $H$ being the maximum time slot. Moreover, we introduce two matrices to manipulate the selection of the different components of $u_{i}$. For all $i \in \mathcal{N}$, define

$$
\begin{aligned}
S_{i}^{\mathrm{mg}} & :=I_{H} \otimes a_{n_{i}, 3}^{\top}, \\
S_{(i, j)}^{\mathrm{tr}} & :=I_{H} \otimes a_{n_{i}, \mathrm{r}(i, j)}^{\top}, \quad \forall j \in \mathcal{N}_{i},
\end{aligned}
$$

where $a_{n_{i}, \ell} \in \mathbb{R}^{n_{i}}$ is a column vector of dimension $n_{i}$ whose entries are all zero except for the $\ell$-th being 1 , and $\mathrm{r}(i, j)$ denotes the index of the variable $p_{(i, j), h}^{\mathrm{tr}}$ in the vector $u_{i, h}$. Thus, $S_{i}^{\mathrm{mg}}$ selects the components of $u_{i}$ associated with the trading with the main grid, while $S_{(i, j)}^{\mathrm{tr}}$ selects the components associated with the trading with agent $j$, i.e.,

$$
\begin{aligned}
S_{i}^{\mathrm{mg}} u_{i} & =\operatorname{col}\left(\left\{p_{i, h}^{\mathrm{mg}}\right\}_{h \in \mathcal{H}}\right)=: p_{i}^{\mathrm{mg}}, \\
S_{(i, j)}^{\mathrm{tr}} u_{i} & =\operatorname{col}\left(\left\{p_{(i, j), h}^{\mathrm{tr}}\right\}_{h \in \mathcal{H}}\right)=: p_{(i, j)}^{\mathrm{tr}}, \quad \forall j \in \mathcal{N}_{i} .
\end{aligned}
$$

Overall, the goal of each agent is to find a strategy $u_{i}^{\star}$ that minimizes its local objective function subject to all the constraints for all time slots, i.e.,

$$
u_{i}^{*} \in \begin{cases}\arg \min _{u_{i}} & J_{i}\left(u_{i}, \sum_{j \in \mathcal{N}} S_{j}^{\mathrm{mg}} u_{j}\right) \\ \text { s.t. } \quad & u_{i} \in \mathcal{U}_{i}:=\prod_{h \in \mathcal{H}} \mathcal{U}_{i, h}, \\ & S_{(i, j)}^{\mathrm{tr}} u_{i}+S_{(j, i)}^{\mathrm{tr}} u_{j}=\mathbf{0}, \forall j \in \mathcal{N}_{i}, \\ \underline{p}^{\mathrm{mg}} \mathbf{1}_{H} \leq \sum_{j \in \mathcal{N}} S_{j}^{\mathrm{mg}} u_{j} \leq \bar{p}^{\mathrm{mg}} \mathbf{1}_{H},\end{cases}
$$

where $J_{i}$ is the cumulative local objective function defined as the summation of (2), (4), (6), and (10) over the horizon $\mathcal{H}$, i.e.,

$$
\begin{aligned}
& J_{i}\left(u_{i}, \hat{p}^{\mathrm{mg}}\right)=\sum_{h \in \mathcal{H}}\left(f_{i, h}^{\mathrm{dg}}\left(p_{i, h}^{\mathrm{dg}}\right)+f_{i, h}^{\mathrm{st}}\left(p_{i, h}^{\mathrm{st}}\right)\right. \\
& \left.\quad+f_{i, h}^{\mathrm{tr}}\left(\left\{p_{(i, j), h}^{\mathrm{tr}}\right\}_{j \in \mathcal{N}_{i}}\right)+f_{i, h}^{\mathrm{mg}}\left(p_{i, h}, \hat{p}_{h}^{\mathrm{mg}}\right)\right),
\end{aligned}
$$

with $\hat{p}^{\mathrm{mg}}=\operatorname{col}\left(\hat{p}_{1}^{\mathrm{mg}}, \ldots, \hat{p}_{H}^{\mathrm{mg}}\right)$, and $\hat{p}_{h}^{\mathrm{mg}}$ as in (9), and (15c)-(15d) represent, in a more compact form, the reciprocity (8) and grid constraints (11), respectively. 


\section{A DISTRIBUTED ALGORITHM FOR EQUILIBRIUM SEEKING IN P2P ENERGY MARKETS}

Each local minimization problem in (15) is coupled with the decisions of the other prosumers both in the cost function (15a) and the constraints (15c)-(15d). Specifically, the coupling enters in the cost associated to the trading with the main grid (10) (in aggregative form), in the reciprocity (8) and in grid constraints (11).

From a game-theoretic perspective, the $N$ inter-dependent optimization problems in (15) define a generalized aggregative game and a set of decisions (or strategies) $u_{1}^{*}, \ldots, u_{N}^{*}$ that simultaneously satisfy (15), for all $i \in \mathcal{N}$, corresponds to a generalized Nash equilibrium (GNE) [8, Def. 1]. In other words, a set of strategies $u_{1}^{*}, \ldots, u_{N}^{*}$ is a GNE if no prosumer (or agent) $i$ can improve its objective function $J_{i}\left(u_{i}^{*}, S_{i}^{\mathrm{mg}} u_{i}^{*}+\sum_{j \neq i}^{N} S_{j}^{\mathrm{mg}} u_{j}^{*}\right)$ by unilaterally changing its strategy $u_{i}^{*}$ to another feasible one. Since the cost functions (15a) are continuous and the constraints (15b)-(15d) are convex and compact, the existence of a GNE follows from Brouwer's fixed-point theorem [21, Prop. 12.7], while uniqueness does not hold in general.

\section{A. A distributed algorithm for GNE seeking}

Several algorithms are available in the literature to find a solution of the aggregative game in (15), e.g. [7]-[10]. Among these methods, the algorithm proposed in [9] for the special class of generalized games with linearly coupled cost functions is shown to be particularly efficient in terms of convergence speed. Here, we propose an upgraded version of [9, Algorithm 1] for the dispatch problem described in (15) capable of treating the coupling (equality) reciprocity constraints in a fully-scalable fashion.

Prior to presenting the proposed distributed algorithm, first, we introduce $\mu_{(i, j)} \in \mathbb{R}^{H}$, for all $j \in \mathcal{N}_{i}$ and $i \in \mathcal{N}$, which denote the dual variable associated with the reciprocity constraints (8), $w_{i} \in \mathbb{R}^{2 H}$ and $\lambda_{i} \in \mathbb{R}^{2 H}$, for all $i \in \mathcal{N}$, which denote an auxiliary and the dual variables, respectively, associated with the grid constraints (11). Furthermore, let us also introduce the step sizes used in the proposed algorithm and formalize their choices.

Assumption 2 (Step size selection): For all $i \in \mathcal{N}$, set

$$
\bar{\alpha}_{i}=\operatorname{blkdiag}\left(\bar{\alpha}_{i, 1}, \ldots, \bar{\alpha}_{i, H}\right),
$$

where $\bar{\alpha}_{i, h}=\operatorname{diag}\left(\alpha_{i, h}^{\mathrm{dg}}, \alpha_{i, h}^{\mathrm{st}}, \alpha_{i, h}^{\mathrm{mg}},\left\{\alpha_{(i, j), h}^{\mathrm{tr}}\right\}_{j \in \mathcal{N}_{i}}\right)$, $\forall h \in \mathcal{H}$, with $\alpha_{i, h}^{\mathrm{dg}}, \alpha_{i, h}^{\mathrm{st}}>0$ and $\alpha_{(i, j), h}^{\mathrm{tr}}>\left|\mathcal{N}_{i}\right|, \forall j \in \mathcal{N}_{i}$; $\beta_{(i, j)}=\beta<\frac{1}{2}, \forall j \in \mathcal{N}_{i} ; \quad \gamma_{i}=\gamma<\frac{1}{2} ; \quad \delta_{i}<\frac{1}{\left|\mathcal{N}_{i}\right|+1}$.

The proposed method is summarized in Algorithm 1. At each stage $k \in \mathbb{N}$, Algorithm 1 works as follows:

1) Strategy update: For all $i \in \mathcal{N}$, prosumer $i$ updates the local decision to $u_{i}(k+1)$ by solving a strongly convex, quadratic programming with linear constraints, for which efficient solvers are available.
Algorithm 1 GNE seeking for P2P Energy Markets

Initialization: For all $i \in \mathcal{N}$, set locally:

(a) Initial conditions: $u_{i}(0) \in \mathcal{U}_{i}, \mu_{(i, j)}(0)=\mathbf{0}$ for all $j \in$ $\mathcal{N}_{i}, w_{i}(0)=\mathbf{0}$ and $\lambda_{i}(0) \in \mathbb{R}_{>0}^{2 H}$.

(b) Step-sizes: $\bar{\alpha}_{i},\left\{\beta_{i j}\right\}_{j \in \mathcal{N}_{i}}, \gamma_{i}$ and $\delta_{i}$ as in Assumption 2.

\section{Iterate until convergence:}

For all $i \in \mathcal{N}$ :

(1) Strategy update:

$$
\begin{array}{r}
u_{i}(k+1)=\arg \min _{z \in \mathcal{U}_{i}}\left\{J_{i}\left(z, \hat{p}^{\mathrm{mg}}(k)+S_{i}^{\mathrm{mg}} u_{i}(k)\right)\right. \\
+\lambda_{i}(k)^{\top}\left[\begin{array}{c}
S_{i}^{\mathrm{mg}} \\
-S_{i}^{\mathrm{mg}}
\end{array}\right] z+\sum_{j \in \mathcal{N}_{i}}\left(\mu_{(i, j)}(k)^{\top} S_{(i, j)}^{\mathrm{tr}} z\right) \\
\left.+\frac{1}{2}\left\|z-u_{i}(k)\right\|_{\bar{\alpha}_{i}}^{2}\right\} .
\end{array}
$$

(2) Communication with neighboring agents:

$$
\begin{array}{lcll}
\text { (a) } & \lambda_{i}(k) & \longrightarrow & \mathcal{N}_{i}, \\
\text { (b) } \quad S_{(i, j)}^{\mathrm{tr}} u_{i}(k+1) & \longrightarrow j, \quad \forall j \in \mathcal{N}_{i} .
\end{array}
$$

(3) Dual variables (reciprocity constraints) update, $\forall j \in \mathcal{N}_{i}$ :

$$
\begin{aligned}
\mu_{(i, j)}(k+1)= & \mu_{(i, j)}(k)-\beta_{i j}\left(S_{(i, j)}^{\mathrm{tr}} u_{i}(k)+S_{(j, i)}^{\mathrm{tr}} u_{j}(k)\right. \\
& \left.-2 S_{(i, j)}^{\mathrm{tr}} u_{i}(k+1)-2 S_{(j, i)}^{\mathrm{tr}} u_{j}(k+1)\right) .
\end{aligned}
$$

(4) Auxiliary variable update:

$$
w_{i}(k+1)=w_{i}(k)+\gamma_{i}\left(\left|\mathcal{N}_{i}\right| \lambda_{i}(k)-\sum_{j \in \mathcal{N}_{i}} \lambda_{j}(k)\right) .
$$

(5) Dual variable (grid constraints) update:

$$
\begin{gathered}
\lambda_{i}(k+1)=\operatorname{proj}_{\mathbb{R}_{\geq 0}^{2 H}}\left(\lambda_{i}(k)+\delta_{i}\left(2\left[\begin{array}{c}
S_{i}^{\mathrm{mg}} \\
-S_{i}^{\mathrm{mg}}
\end{array}\right] u_{i}(k+1)\right.\right. \\
\left.\left.-\left[\begin{array}{c}
S_{i}^{\mathrm{mg}} \\
-S_{i}^{\mathrm{mg}}
\end{array}\right] u_{i}(k)-\left[\begin{array}{c}
\frac{\bar{p}^{\mathrm{mg}}}{N} \mathbf{1}_{H} \\
-\frac{p^{\mathrm{mg}}}{N} \mathbf{1}_{H}
\end{array}\right]-2 w_{i}(k+1)+w_{i}(k)\right)\right) .
\end{gathered}
$$

2) Communication: For all $i \in \mathcal{N}$, prosumer $i$ sends

a) the most recent value of its local copy of the dual variable, $\lambda_{i}(k)$, to all its neighbors;

b) the most recent value of its trading strategy, $S_{(i, j)}^{\mathrm{tr}} u_{i}(k)$, to the corresponding trading partner $j \in \mathcal{N}_{i}$.

3) Dual variable (reciprocity constraints) update: For all $i$, prosumer $i$ updates the local copy of the dual variable $\mu_{(i, j)}$ exploiting the most recent value of its own trading strategy, $S_{(i, j)}^{\mathrm{tr}} u_{i}(k)$, and that of the trading partner, $S_{(j, i)}^{\mathrm{tr}} u_{j}(k)$.

4) Auxiliary variable update: For all $i \in \mathcal{N}$, prosumer $i$ updates the auxiliary variable $w_{i}$ according to a discrete-time integration dynamics driven by the disagreement on the local dual variables associated with the grid constraints.

5) Dual variable (grid constraints) update: For all $i \in \mathcal{N}$, prosumer $i$ updates the local copy of the dual variable $\lambda_{i}$ according to a projected-gradient ascent combined with a discrete integral dynamics driven by the disagreement on the dual variables. 
Remark 1 (Algorithm 1):

(i) The choice of the step sizes (Assumption 2) of each prosumer is based on local information only. In particular, there is no need for the prosumers to know the global properties of the P2P market.

(ii) We assume that each prosumer has a perfect knowledge of the aggregate load on the grid $\hat{p}^{\mathrm{mg}}(k)$, at each iteration $k$ of the algorithm. In practice, the value of $\hat{p}^{\mathrm{mg}}$ can be broadcast to the prosumers by a central coordinator, thus preserving the scalability of the algorithm.

(iii) In step (1), the second argument of $J_{i}$ reads as $\hat{p}^{\mathrm{mg}}(k)+$ $S_{i}^{\mathrm{mg}} u_{i}(k)$, where $S_{i}^{\mathrm{mg}} u_{i}(k)$ is a correction term due to the contribution of agent $i$ to the aggregate load $\hat{p}^{\mathrm{mg}}$. When the correction term is not considered, Algorithm 1 converges to a generalized aggregative (or Wardrop) equilibrium (GAE) [8, Def. 2].

In the next statement, we prove global convergence of Algorithm 1 to a (variational) GNE of the game in (15).

Proposition 1 (Convergence): The sequence of collective strategies $\left(\left(u_{1}(k), \ldots, u_{N}(k)\right)_{k \in \mathbb{N}}\right.$ generated by Algorithm 1 converges to a (variational) GNE of the game in (15).

Proof (sketch): Due to space limitations, we provide only a sketch of the two main steps of the proof:

S.1 The iterations in Algorithm 1 are obtained by applying the distributed method in [9, Algorithm 1] on the economic dispatch problem presented in Section II-B.

S.1 The game in (15) satisfies all the technical assumptions in [9, Theorem 1], that proves global convergence of [9, Algorithm 1] to a (variational) GNE.

\section{Numerical Results}

This section presents simulation-based tests of the considered hybrid P2P market. In order to perform a simulation, first, a test case is synthesized and then the game in (15), which is based on the test case and where $K=24$, for each $i \in \mathcal{N}$, is solved by Algorithm 1. The stopping criteria in the simulations use the primal and dual residuals associated with the reciprocity constraints [17], i.e., $\left\|\operatorname{col}\left(\left\{S_{(i, j)}^{\operatorname{tr}} u_{i}(k)+S_{(j, i)}^{\operatorname{tr}} u_{j}(k)\right\}_{j \in \mathcal{N}_{i}, i \in \mathcal{N}}\right)\right\|_{2} \leq 0.01$ and $\left\|\operatorname{col}\left(\left\{u_{i}(k+1)-u_{i}(k)\right\}_{i \in \mathcal{N}}\right)\right\|_{2} \leq 0.1$, respectively.

In each test case, we consider a heterogeneous network, which consists of three types of prosumers (agents), i.e., small-scale residential, large-scale residential, and industrial. The differences among these three types are their loads, particularly, their maximum values and their daily profiles, and the scale of their dispatchable generators and storage units, if available. Furthermore, the per-unit costs of the power production from the dispatchable generators differ based on the type of prosumer, where industrial prosumers have the smallest per-unit cost and small-scale residential prosumers have the largest one. We also set $q_{h}^{\mathrm{mg}}=N^{-1}$, for all $h \in \mathcal{H}$. Moreover, $q_{i}^{\text {st }}=c_{i}^{\text {st }}=0$, for all $i \in \mathcal{N}^{\text {st }}$, and $c_{(i, j)}^{\mathrm{tr}}=1$, for all $j \in \mathcal{N}_{i}$ and $i \in \mathcal{N}$.

\section{A. Power generation profile}

The first aspect that we evaluate is the advantage of having storage units, which influence the power generation
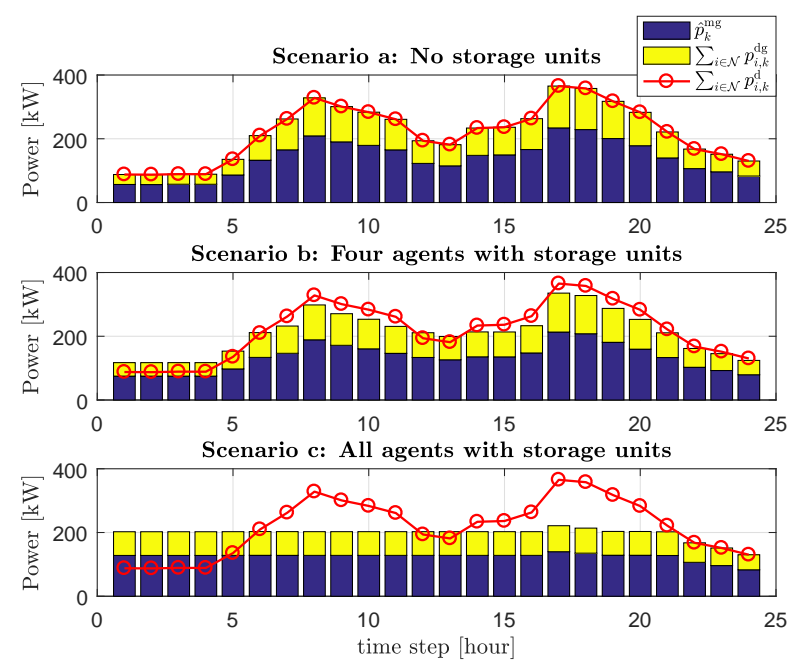

Fig. 1. Total power generation from dispatchable generation units and importing from the main grid vs. total loads in a 10-agent network.
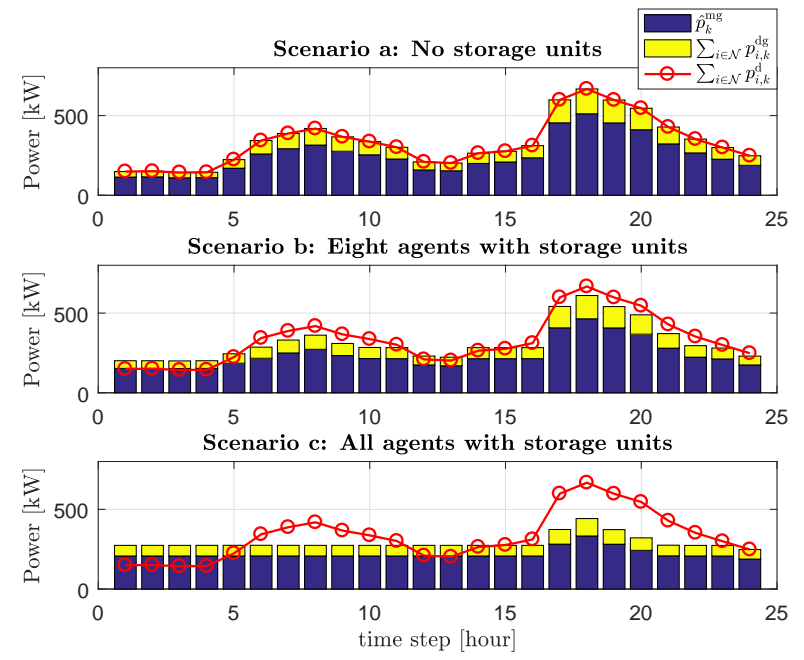

Fig. 2. Total power generation from dispatchable generation units and importing from the main grid vs. total loads in a 20 -agent network.

profile of the network. Therefore, two networks, one with 10 agents and the other with 20 agents, are randomly generated. Each agent has a dispatchable generation unit. Moreover, we consider three scenarios, as follows: a. all agents do not have storage units, b. some of the agents have storage units, and c. all agents have storage units.

The simulation results are shown in Figures 1-2, which depict the total load and total generation profiles of each network and Table I. As can be seen, when the storage units are introduced to the network, the total power generation profile is different from that of the load. We can observe that in scenario $\mathrm{c}$, the generation profile is relatively flat. By having storage units, the agents can produce and buy energy from the main grid during the off-peak hours (e.g., at the $1^{\text {st }}-4^{\text {th }}$ hours) and use it during the peak hours (e.g., at the $15^{\text {th }}-20^{\text {th }}$ hours), resulting in a significant cost reduction (see Table I). Note that, in each simulation, the aggregate load to the main grid, $\hat{p}_{h}^{\mathrm{mg}}$, for each $h \in \mathcal{H}$, satisfies (11). 
TABLE I

TOTAL COST IN SECTION IV-A (PROPORTIONAL)

\begin{tabular}{cccc}
\hline Scenario & a & b & c \\
\hline 10-agent network & $100 \%$ & $-11.7 \%$ & $-27.6 \%$ \\
20-agent network & $100 \%$ & $-14.5 \%$ & $-33.7 \%$ \\
\hline
\end{tabular}

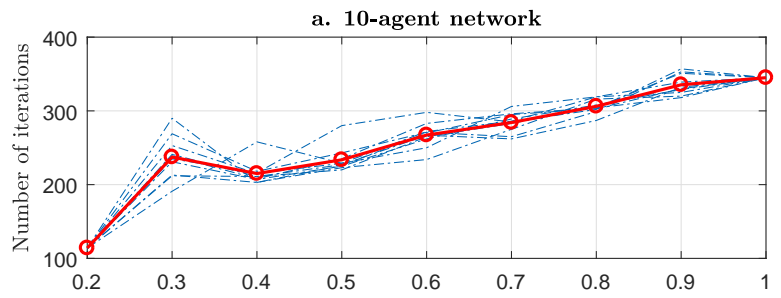

b. 20-agent network

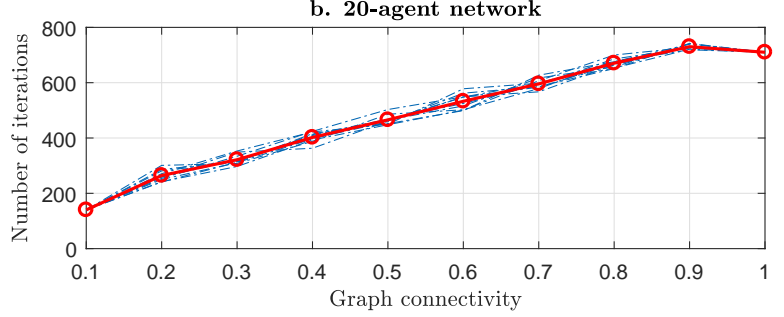

Fig. 3. Graph connectivity vs. number of iterations. Blue dash-dotted lines indicate the simulation data and red circles indicate the mean values.

\section{B. Sparsity effect}

In the second simulation study, the effect of network sparsity on the number of iterations performed is evaluated. Hence, a network of 10 agents and that of 20 agents are randomly generated with different levels of graph connectivity. Note that the connectivity level ranges from $0(\mathcal{E}=\emptyset)$ to 1 (a complete graph). For the simulations of the 10agent network, the graph connectivity level varies from 0.2 to 1 , with 0.1 step, whereas for the simulations of the 20 -agent network, it varies from 0.1 to 1 , with 0.1 step. At each connectivity level, 10 Monte Carlo simulations, where the links are generated randomly, are carried out. The results of these simulations are presented in Figure 3. It can be observed that the number of iterations required increases as the network connectivity increases. This result is expected since the number of coupling constraints that must be satisfied grows with the addition of links in the network.

\section{CONCLUSION}

In a hybrid peer-to-peer energy market model, where prosumers can trade among themselves and with the main grid, the economic dispatch problem can be modeled as a noncooperative game with coupling constraints. We propose a fully-scalable and distributed algorithm to steer the system to a generalized Nash equilibrium. Numerical studies, conducted in scenarios with real data, show that the proposed energy management approach has the potential to ensure a safe and efficient operation of the electrical power grid, namely, by reducing the individual economic expenses, while respecting the reciprocity of the tradings and the limits on the aggregate load.

\section{REFERENCES}

[1] W. Tushar, C. Yuen, H. Mohsenian-Rad, T. Saha, H. V. Poor, and K. L. Wood, "Transforming energy networks via peer-to-peer energy trading: The potential of game-theoretic approaches," IEEE Signal Processing Magazine, vol. 35, no. 4, pp. 90-111, 2018.

[2] F. Dörfler, S. Bolognani, J. W. Simpson-Porco, and S. Grammatico, "Distributed control and optimization for autonomous power grids," in 2019 18th European Control Conference (ECC). IEEE, 2019, pp. 2436-2453.

[3] W. Saad, Z. Han, H. Poor, and T. Başar, "Game theoretic methods for the smart grid," IEEE Signal Processing Magazine, pp. 86-105, 2012.

[4] A.-H. Mohsenian-Rad, V. W. Wong, J. Jatskevich, R. Schober, and A. Leon-Garcia, "Autonomous demand-side management based on game-theoretic energy consumption scheduling for the future smart grid," IEEE Transactions on Smart Grid, vol. 1, no. 3, pp. 320-331, 2010.

[5] I. Atzeni, L. G. Ordóñez, G. Scutari, D. P. Palomar, and J. R. Fonollosa, "Demand-side management via distributed energy generation and storage optimization," IEEE Transactions on Smart Grid, vol. 4, no. 2, pp. 866-876, 2012.

[6] G. Belgioioso and S. Grammatico, "Semi-decentralized Nash equilibrium seeking in aggregative games with coupling constraints and nondifferentiable cost functions," IEEE Control Systems Letters, vol. 1, no. 2, pp. 400-405, 2017.

[7] D. Paccagnan, B. Gentile, F. Parise, M. Kamgarpour, and J. Lygeros, "Nash and Wardrop equilibria in aggregative games with coupling constraints," IEEE Transactions on Automatic Control, vol. 64, no. 4, pp. 1373-1388, 2018.

[8] G. Belgioioso and S. Grammatico, "A Douglas-Rachford splitting for semi-decentralized equilibrium seeking in generalized aggregative games," in Proceedings of the IEEE Conference on Decision and Control (CDC), Miami, USA, 2018, pp. 3541-3546.

[9] — "A distributed proximal-point algorithm for Nash equilibrium seeking in generalized potential games with linearly coupled cost functions," in Proceedings of the IEEE European Control Conference, Naples, Italy, 2019, DOI: 10.23919/ECC.2019.8795852.

[10] P. Yi and L. Pavel, "An operator splitting approach for distributed generalized Nash equilibria computation," Automatica, vol. 102, pp. 111-121, 2019.

[11] J. Barreiro-Gomez, G. Obando, and N. Quijano, "Distributed population dynamics: Optimization and control applications," IEEE Transactions on Systems, Man, and Cybernetics: Systems, vol. 47, no. 2, pp. 304-314, 2017.

[12] J. Hu, R. Harmsen, W. Crijns-Graus, E. Worrell, and M. van den Broek, "Identifying barriers to large-scale integration of variable renewable electricity into the electricity market: A literature review of market design," Renewable and Sustainable Energy Reviews, vol. 81, pp. 2181-2195, 2018.

[13] T. Sousa, T. Soares, P. Pinson, F. Moret, T. Baroche, and E. Sorin, "Peer-to-peer and community-based markets: A comprehensive review," Renewable and Sustainable Energy Reviews, vol. 104, pp. 367378, 2019.

[14] — , "Peer-to-peer and community-based markets: A comprehensive review," Renewable and Sustainable Energy Reviews, vol. 104, pp. 367-378, 2019.

[15] Y. Parag and B. K. Sovacool, "Electricity market design for the prosumer era," Nature Energy, vol. 2, no. 1603, 2016.

[16] T. Morstyn, A. Teytelboym, and M. D. McCulloch, "Bilateral contract networks for peer-to-peer energy trading," IEEE Transactions on Smart Grid, vol. 10, no. 2, pp. 2026-2035, 2018.

[17] T. Baroche, F. Moret, and P. Pinson, "Prosumer markets: A unified formulation," in Proceedings of IEEE PowerTech Conference, Milano, Italy, 2019, DOI: 10.1109/PTC.2019.8810474.

[18] C. A. Hans, P. Braun, J. Raisch, L. Grüne, and C. Reincke-Collon, "Hierarchical distributed model predictive control of interconnected microgrids," IEEE Transactions on Sustainable Energy, vol. 10, no. 1, pp. 407-416, 2019.

[19] E. Sorin, L. Bobo, and P. Pinson, "Consensus-based approach to peer-to-peer electricity markets with product differentiation," IEEE Transactions on Power Systems, vol. 34, no. 2, pp. 994-1004, 2019.

[20] H. L. Cadre, P. Jacquot, C. Wan, and C. Alasseur, "Peer-to-peer electricity market analysis: From variational to generalized Nash equilibrium," European Journal of Operational Research, vol. 282, no. 2, pp. 753-771, 2020.

[21] D. P. Palomar and Y. C. Eldar, Convex optimization in signal processing and communications. Cambridge university press, 2010. 